\title{
Intraoperative ureter visualization using a near-infrared imaging agent
}

Richard W. Farnam

Richard G. Arms, III

Alwin H. Klaassen

Jonathan M. Sorger 


\title{
Intraoperative ureter visualization using a near-infrared imaging agent
}

\author{
Richard W. Farnam, ${ }^{a}$ Richard G. Arms III, ${ }^{b}$ Alwin H. Klaassen, ${ }^{c}$ and Jonathan M. Sorger ${ }^{c, \star}$ \\ aTexas Urogynecology and Laser Surgery Center, Las Palmas Medical Center, El Paso, Texas, United States \\ ${ }^{b}$ Texas Tech University Health Sciences Center, Department of Obstetrics and Gynecology, El Paso, Texas, United States \\ 'Intuitive Surgical, Sunnyvale, California, United States
}

\begin{abstract}
The fluorescent imaging agent IS-001 was determined to be well tolerated in all subjects and has the potential to provide ureter visualization throughout minimally invasive hysterectomy procedures. This study was conducted to evaluate clinical safety and efficacy of a real-time ureter visualization technique for use during hysterectomy surgery. The study drug appears safe, is renally excreted, and allows enhanced ureter visualization when imaged with a clinically approved near-infrared sensitive endoscope. This is a first-in-human study showing preliminary results that the drug is safe and effective during surgery for improved ureter visualization. () The Authors. Published by SPIE under a Creative Commons Attribution 4.0 Unported License. Distribution or reproduction of this work in whole or in part requires full attribution of the original publication, including its DOI. [DOI: 10.1117/1.JBO.24.6.066004]
\end{abstract}

Keywords: ureter imaging; robotic-assisted minimally invasive surgery; near-infrared; hysterectomy.

Paper 190091RR received Mar. 29, 2019; accepted for publication May 17, 2019; published online Jun. 18, 2019.

\section{Introduction}

Ureteral injury is a serious complication of gynecological and colorectal surgery that frequently goes unrecognized intraoperatively. ${ }^{1}$ Iatrogenic ureteral injury occurs during laparoscopic gynecologic surgery with an incidence of $0.3 \%$ to $2.5 \%^{2}$ with injury rates for high-risk reconstructive pelvic surgeries up to $11 \% .^{3}$ Only about one-third of ureteral injuries are detected intraoperatively, leading to delayed diagnosis and treatment with deleterious consequences for the patient. ${ }^{4}$ Iatrogenic ureteral injury imposes a significant burden in terms of morbidity and increased health care costs and represents a medicolegal challenge for physicians. Sequelae can include fistula and loss of the affected kidney. Risk factors for ureteral injury include the ureter's close proximity to the gynecologic organs within the pelvis, distortion of normal anatomic relationships by pathology such as endometriosis, and surgeon experience. ${ }^{5}$ Avoidance of ureteral injury depends upon clear understanding of anatomic relationships and meticulous surgical technique, including careful dissection of pelvic structures. ${ }^{6}$

Minimally invasive surgery (MIS) offers several advantages over traditional open surgical techniques including reduced infection rates, shorter hospital stays, and rapid return to normal activities $^{7}$ and is becoming an increasingly more common approach for hysterectomy. ${ }^{8}$ One potential drawback to all MIS approaches is an increased risk of inadvertent ureteral injury ${ }^{7,9}$ when compared to open techniques.

A variety of renally excreted dyes have been administered in both the preclinical and clinical setting over the past four decades with the goal of ureteral visualization. Indigo carmine, ${ }^{10}$ sodium fluorescein, ${ }^{11}$ and methylene blue ${ }^{12,13}$ have been explored by several groups for use in humans, and a variety of experimental dyes ${ }^{14-18}$ have been used in preclinical studies.

Intraoperative near-infrared (NIR) fluorescence imaging is a promising technique that offers real-time visual information

*Address all correspondence to Jonathan Sorger, E-mail: jonathan.sorger@ intusurg.com about tissues and structures by utilizing wavelengths not visible to the naked eye. One advantage of this in the surgical setting is that visualization of normal tissue is not altered, as is the case with blue dyes and fluorescein. NIR fluorescence imaging in conjunction with the fluorescent dye IS-001 has the potential to provide contrast for improved ureter visualization. In addition, the excitation (peak $\sim 780 \mathrm{~nm}$ ) and emission (peak $\sim 815 \mathrm{~nm}$ ) spectra of IS-001 are compatible with clinically available robotic and laparoscopic imaging systems.

\section{Study Design and Objectives}

The clinical study was performed at Las Palmas Medical Center and the Texas Urogynecology and Laser Surgery Center (El Paso, Texas) between February 2, 2017, and September 9, 2017. All study procedures were reviewed and approved by the Las Palmas Del Sol Healthcare Institutional Review Board (IRB) and conducted under an Investigational New Drug (IND) application with the United States Food and Drug Agency (USFDA) in compliance with Good Clinical Practice (GCP). Signed informed consent was received from all subjects prior to initiation of any clinical study procedure.

This clinical study was designed as a single site, open-label, nonrandomized, dose-escalating study enrolling 24 women aged 18 to 65 undergoing robotic-assisted minimally invasive hysterectomy. Study sample size was based on historical norms for standard phase- 1 clinical safety trials. The primary objective of this clinical study was to assess safety and patient tolerance of intravenously (IV) injected IS-001 investigational drug on subjects undergoing robotic hysterectomy. The secondary objective was to evaluate the blood plasma drug pharmacokinetic parameters following IV injection. An additional exploratory objective involved the intraoperative assessment of ureter visibility, fluorescence intensity, and duration.

\section{Safety Evaluations and Study Procedures}

Subjects were recruited for the study from the investigator's clinical practice and evaluated against the study inclusion and 
Table 1 Inclusion/exclusion criteria.

Study participant criteria

Inclusion criteria

1. Subject is between the ages of 18 and 65

2. Subject is scheduled to undergo robotic hysterectomy using a da Vinc $^{\circledR} \mathrm{Si} / \mathrm{Xi}$ surgical system with Firefly ${ }^{\circledR}$ fluorescent imaging

3. Subject is willing and able to provide informed consent

4. Subject is considered capable of complying with study procedures

5. Subject has no medical history of liver or kidney disease

6. Subject has no evidence of NYHA classes II to IV cardiac disease

7. Subject has recent (<3 months) clinical hematology $(C B C)$ values within the acceptable values reference range [WBC $\left(3.5\right.$ to $\left.10.5 \mathrm{~K} / \mathrm{mm}^{3}\right)$ and platelet count $\left(150\right.$ to $\left.450 \mathrm{~K} / \mathrm{mm}^{3}\right)$ ]

8. Subject has recent (<3 months) clinical serum chemistry (CMP) values within the acceptable values reference range [eGFR (>60 mL/min $/ 1.73 \mathrm{~m}^{2}$ ), ALT (7 to $55 \mathrm{U} / \mathrm{L}$ ), AST (5 to $40 \mathrm{U} / \mathrm{L}$ ), ALP (39 to $118 \mathrm{U} / \mathrm{L})$, and total serum bilirubin $(0.1$ to $1.2 \mathrm{mg} / \mathrm{dL})$ ]

Exclusion criteria

1. Subject is pregnant or nursing

2. Subject has a history of alcoholism

3. Subject has a history of drug abuse

4. Subject has known active hepatitis $B$ virus (HBV) or hepatitis $C$ virus (HCV) infection

5. Subject has known human immunodeficiency virus (HIV) infection

6. Subject has been diagnosed with or treated for cancer in the last 2 years

7. Subject has a total body weight $<32 \mathrm{~kg}$

8. Subject has after $5 \mathrm{~min}$ of supine rest a diastolic blood pressure $\geq 100 \mathrm{mmHg}$ and/or a systolic blood pressure $\geq 160 \mathrm{mmHg}$

9. Subject has after 5 min of supine rest a resting heart rate $\leq 35$ or $\geq 115 \mathrm{bpm}$

10. Subject has any other condition or personal circumstance that, in the judgment of the investigator, might interfere with the collection of complete good quality data or represents an unacceptable safety liability

exclusion criteria (Table 1). Participants were selected as those scheduled to undergo hysterectomy using a $d a$ Vinci $^{\circledR} \mathrm{Si}$ or Xi surgical system with Firefly ${ }^{\circledR}$ fluorescent imaging for a benign condition. Study procedures followed from a three visit schedule (Table 2), with screening and baseline evaluations [vital signs, 12-lead electrocardiogram (ECG), serum chemistry, serum hematology, and urinalysis (UA)] conducted on visit 1 within $\sim 72$-h prior to study drug administration. Visit 2 consisted of 2 days, with hysterectomy and investigational drug administration on day 1 , followed by a 24 -h postinjection follow-up on day 2 . The investigational imaging agent was administered IV as a slow-bolus injection over the course of $1 \mathrm{~min}$. Postdrug 12lead ECG, serum chemistry, and hematology were performed at $\sim 6$-h postinjection on day 1 . Intraoperative vital signs were recorded pre- and postinjection. Periodic blood samples were collected preinjection, at $2,10,30$, and $60 \mathrm{~min}$ in addition to 2, 4, and $6 \mathrm{~h}$ postinjection for pharmacokinetic drug-plasma analysis. Intraoperative ureter fluorescence visualization observations were made at 10, 30, and $60 \mathrm{~min}$ (or last possible timepoint if surgery lasted less than $60 \mathrm{~min}$ ) postinjection. Images in Firefly ${ }^{\circledR}$ were assessed by the investigator intraoperatively for ureter fluorescence intensity scored on a 4-point scale from 0 to 3 , where $0=$ none, $1=$ mild, $2=$ moderate, and $3=$ strong fluorescence of the ureter. On day 2 of visit 2, at 24-h follow-up, additional postinjection serum chemistry, serum hematology, and UA samples were analyzed and vital signs were recorded. At visit 3 (14 days \pm 3 days postinjection), a follow-up consisting of serum chemistry, serum hematology, and UA was performed, and vital signs were recorded. Treatment emergent adverse events were monitored from postinjection through the $14 \pm 3$ days follow-up until study completion. Safety results were evaluated as shifts from baseline to postinjection and shifts outside the normal reference range. Safety evaluations were tabulated, and based on incidence, clinical significance, and changes in laboratory results but were not statistically powered to detect differences in safety between groups.

\section{Pharmacokinetic Assessments and Analysis}

Blood samples for pharmacokinetic analysis were collected in potassium EDTA collection tubes. After, blood collection samples were kept on ice until centrifugation. Within $60 \mathrm{~min}$ of collection, samples were centrifuged at $3000 \times \mathrm{g}$ for $10 \mathrm{~min}$ at $4^{\circ} \mathrm{C}$, the plasma harvested and aliquoted into plastic tubes, and immediately frozen at $-80^{\circ} \mathrm{C}$ until shipped on dry ice to the central analytical laboratory. Drug-plasma concentrations were analyzed by validated high-performance liquid chromatography and tandem mass spectrometry (LC-MS/MS) assay with a lower limit of quantitation (LLOQ) at $0.91 \mathrm{ng} / \mathrm{ml}$. The IS-001 plasma concentration-time data for each subject were analyzed by noncompartmental methods using Phoenix WinNonLin ${ }^{\circledR}$ version 6.2 (Pharsight Corp., Mountain View, California). The noncompartmental analysis provided estimates of the following parameters: plasma concentration at 2 min following the start of the IS-001 IV infusion $\left(C_{2-\min }\right)$ obtained by log-linear extrapolation of the observed plasma drug concentration-time data, terminal elimination rate constant $\left(\lambda_{z}\right)$ estimated by linear regression of the terminal exponential component of the log IS-001 plasma concentration-time curve, elimination $\left(t_{1 / 2-\lambda z}\right)$ determined by dividing $\ln (2)$ by $\lambda_{z}$, the area under the plasma concentration-time curve from time 0 to infinity $\left(\mathrm{AUC}_{0-\infty}\right)$ obtained by dividing the last observed plasma concentration $\geq$ lower limit of quantitation by $\lambda_{z}$ as the sum of the extrapolated area and $\mathrm{AUC}_{0 \text {-last }}$, clearance $(\mathrm{CL})$ calculated by dividing the dose by $\mathrm{AUC}_{0-\infty}$, and volume of distribution $\left(V_{\lambda z}\right)$ estimated by dividing the CL by $\lambda_{z}$.

\section{Results}

Drug dose assignments followed a sequential, dose escalation design with the first eight subjects receiving a single $10 \mathrm{mg}$ $(n=8)$ IS-001 IV injection, the subsequent eight subjects receiving a single $20 \mathrm{mg}(n=8)$ IS-001 IV injection, and the final eight subjects enrolled receiving a single $40 \mathrm{mg}(n=8)$ 
Table 2 Schedule of events.

\begin{tabular}{|c|c|c|c|c|c|c|c|c|c|c|c|}
\hline \multirow[b]{2}{*}{ Activity } & \multirow{2}{*}{$\begin{array}{l}\text { Visit } 1 \text { screening } \\
\text { and admission }\end{array}$} & \multicolumn{9}{|c|}{ Visit 2 study drug administration } & \multirow{2}{*}{$\begin{array}{c}\begin{array}{c}\text { Visit } 3 \\
\text { follow-up }\end{array} \\
14 \pm 3 \text { days }\end{array}$} \\
\hline & & Pre & $2 \min$ & $10 \mathrm{~min}$ & $30 \mathrm{~min}$ & $60 \mathrm{~min}$ & $2 \mathrm{~h}$ & $4 \mathrm{~h}$ & $6 \mathrm{~h}$ & $24 \mathrm{~h}$ & \\
\hline Demographics & $x$ & & & & & & & & & & \\
\hline Inclusion/exclusion criteria & $x$ & & & & & & & & & & \\
\hline Informed consent & $\mathrm{x}$ & & & & & & & & & & \\
\hline Pregnancy test (if applicable) & $x$ & & & & & & & & & & \\
\hline Vitals signs & $x^{a}$ & $X^{b}$ & & $X^{b}$ & & $\mathrm{X}^{b}$ & & & $x^{a}$ & $x^{a}$ & $x^{a}$ \\
\hline \multicolumn{12}{|c|}{ Cardiology assessment } \\
\hline Electrocardiogram (12-Lead ECG) & $x$ & & & & & & & & $x$ & & \\
\hline \multicolumn{12}{|c|}{ Blood collection procedures } \\
\hline Serum chemistry panel (CMP) & $\mathrm{x}$ & & & & & & & & $\mathrm{x}$ & $x$ & $x$ \\
\hline $\mathrm{CBC}$ & $x$ & & & & & & & & $x$ & $x$ & $\mathrm{x}$ \\
\hline Blood PK sample & & $x$ & $x$ & $x$ & $\mathrm{x}$ & $x$ & $x$ & $x$ & $x$ & & \\
\hline \multicolumn{12}{|c|}{ Urine sample collection procedures } \\
\hline Urine collection (for safety routine UA) & $x$ & & & & & & & & & $x$ & $x$ \\
\hline \multicolumn{12}{|c|}{ Additional assessment } \\
\hline Adverse events monitoring & & $x$ & $x$ & $x$ & $x$ & $x$ & $x$ & $x$ & $\mathrm{x}$ & $x$ & $x$ \\
\hline Recording of concomitant medications & & $\mathrm{x}$ & $x$ & $x$ & $x$ & $x$ & $x$ & $x$ & $\mathrm{x}$ & $\mathrm{x}$ & \\
\hline Fluorescence screen capture & & & & $x$ & $x$ & $x$ & $X^{c}$ & & & & \\
\hline
\end{tabular}

$\mathrm{X}^{a}$ : Awake vital signs (postinjection will be compared to baseline).

$\mathrm{X}^{b}$ : Anesthesia vital signs (postinjection will be compared to baseline).

$\mathrm{X}^{c}$ : At latest possible time-point during surgery.

IS-001 IV injection. Figure 1 summarizes the flow of subjects through the screening process to drug-dose cohort. A minimum of $24 \mathrm{~h}$ separated individual subjects' dosing to allow for appropriate safety evaluation before a new subject was injected. Dose groups were chosen based on the preclinical safety and pharmacodynamics evaluations of IV IS-001 injection. Dose-cohorts were completed in sequential escalation to allow for full safety evaluation of each dose before a subsequent higher dose was administered. The drug dose-cohort groups had similar baseline characteristics. No placebo was injected, and post-treatment results were compared to pretreatment baseline measurements. IS-001 was injected at the beginning of the hysterectomy procedure when the patient was under anesthesia just prior to robotic endoscope insertion into the abdomen.

Participants were monitored for adverse events (AEs) from investigational drug injection on visit 2 through the 14-day follow-up and end of study. Only treatment emergent adverse events not typically associated with hysterectomy surgery or the surgical recovery process were recorded as AEs. A total of three AEs consistent with this categorization were observed in a total of two subjects, both in the lowest drug-dose cohort $(10 \mathrm{mg})$, none of which were deemed drug related. No further adverse events were observed in any other subject or in any of the escalating drug dose-cohorts. The treatment emergent AEs are coded using the Medical Dictionary for Regulatory Activities (MedDRA) and listed in Table 3.

All changes in safety-related laboratory parameters observed were consistent with the underlying hysterectomy surgery being performed during study drug administration and the recovery from surgery. No individual change in laboratory parameters was deemed clinically significant. Notable shifts from baseline are provided in Table S1 in the Supplementary Materials, which also shows the mean change from baseline of white blood cell count (WBC) after drug administration and surgery. An increased WBC is consistent with the hysterectomy surgery and recovery. ${ }^{19,20}$

There was no dose-dependent increase in mean change from baseline for WBC, suggesting this effect was not drug related. Mean change from baseline normalized over time to the 14-day recovery. In addition, Table S1 in the Supplementary Materials shows the mean change from baseline of red blood cell count (RBC), percent hematocrit (HCT), and hemoglobin $(\mathrm{HgB})$. The decrease observed in RBC, HCT, and $\mathrm{HgB}$ is consistent with surgery and recovery. ${ }^{21}$ These values show no dose-dependent increase in mean change from baseline, suggesting the effect was not drug related. The mean changes from baseline 


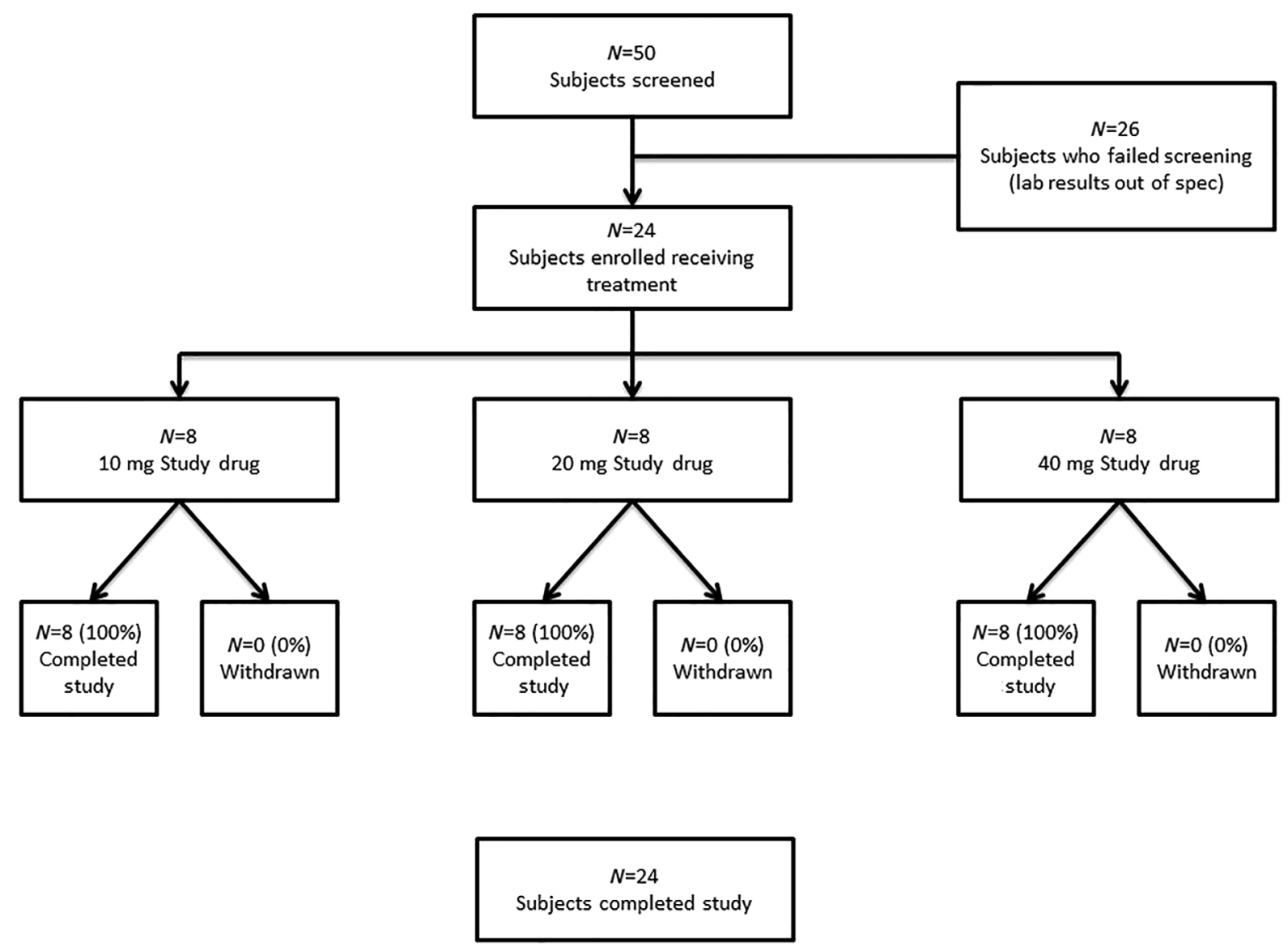

Fig. 1 Disposition of study participants. Of the 50 subjects screened, 26 failed to meet serum chemistry or hematology inclusion criteria. The remaining 24 subjects were assigned to a drug dose-cohort in an escalating fashion based on their enrollment in the study.

normalized over time to the 14-day recovery visit. Changes in serum albumin and calcium were also observed and are shown as mean change from baseline. A decrease in serum albumin ${ }^{22}$ and serum calcium ${ }^{23}$ is consistent with surgery and recovery. These values show no dose-dependent increase in mean change

Table 3 Treatment emergent adverse events by preferred term.

Treatment emergent adverse events by MedDRA ${ }^{a}$ preferred term

Total number of subjects

24

Total number of adverse events

4

Number of subjects (\%) reporting $\geq 1$ treatmentemergent event

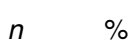

Nervous system disorders > headache (10019211)

Renal and urinary disorders $>$ urinary tract infection (10046571)

Musculoskeletal and connective tissue

disorders > neck pain (10028836)

Injury, poisoning, and procedural complications > medical device site pain (10076133)

aMedical dictionary for regulatory activities. from baseline, suggesting the effect was not drug related. These mean changes from baseline normalized over time to the 14-day recovery visit. Table S2 in the Supplementary Materials shows the laboratory value shifts outside of the normal reference range as fraction of subjects. An additional observed increase was seen in a fraction of participants presenting with occult blood in urinalysis [10 mg-baseline (1/8), $24 \mathrm{~h} \mathrm{(8/8),} 14$ day (4/8), $20 \mathrm{mg}$-baseline (2/8), $24 \mathrm{~h}$ (7/8), 14 day (3/8), $40 \mathrm{mg}$-baseline (4/8), $24 \mathrm{~h}$ (7/8), 14 day (3/8)]. These results are also consistent with hysterectomy surgery and insertion and removal of the foley catheter. $^{24}$ These incidence values show no dosedependent increase, suggesting the effect was not drug related. No other notable change was seen in any other laboratory parameter including 12-lead ECG (QTc) or vital sign measurements.

Six hours after IV administration, drug-plasma levels were at or near the limit of quantitation $(0.91 \mathrm{ng} / \mathrm{ml})$ (Table 4). Pharmacokinetic analysis shows that IS-001 plasma concentrations decline in a biexponential pattern following IV administration. Plasma elimination is rapid with mean terminal half-life $\left(t_{1 / 2-\lambda z} s\right)$ ranging from 0.5 to $2.5 \mathrm{~h}$ (Table 5).

Intravenous injection of IS-001 produced ureter fluorescence when imaged with the $d a$ Vinci $^{\circledR}$ Surgical System's Firefly ${ }^{\circledR}$ fluorescent imaging at all tested doses as shown in Fig. 2. The 40-mg dose-cohort showed the strongest ureter fluorescence at all time-points evaluated postinjection as assessed by the operating surgeon. At $10 \mathrm{~min}$ postinjection, the $40-\mathrm{mg}$ dose-cohort showed the highest fluorescence intensity with ureter images in 
Table 4 IS-001 plasma concentrations.

\begin{tabular}{|c|c|c|c|c|c|c|c|c|c|}
\hline \multirow[b]{2}{*}{ Dose $(\mathrm{mg})$} & \multirow[b]{2}{*}{ Time } & \multicolumn{8}{|c|}{ IS-001 plasma concentrations ( $\mathrm{ng} / \mathrm{mL})$} \\
\hline & & Predose & $2 \min$ & $10 \mathrm{~min}$ & $30 \mathrm{~min}$ & $60 \mathrm{~min}$ & $120 \mathrm{~min}$ & $240 \min$ & $360 \min$ \\
\hline \multirow[t]{4}{*}{10} & Mean & $\mathrm{BQL}^{\mathrm{a}}$ & 2676 & 614 & 95.1 & 39.4 & 12.4 & 2.9 & 2.7 \\
\hline & $C V^{b} \%$ & $\mathrm{BQL}^{\mathrm{a}}$ & $25.6 \%$ & $48.9 \%$ & $55.5 \%$ & $122.2 \%$ & $170.6 \%$ & $127.4 \%$ & $75.8 \%$ \\
\hline & Median & $\mathrm{BQL}^{\mathrm{a}}$ & 2395 & 536 & 92.1 & 20.3 & 4.6 & 1.4 & 1.6 \\
\hline & Range & $B L^{a}$ & 2055 to 3981 & 259 to 1086 & 34.5 to 195 & 13.3 to 156 & 2.0 to 64.4 & 0.9 to 11.7 & $B L^{a}$ to 6.0 \\
\hline \multirow[t]{4}{*}{20} & Mean & $B Q L^{a}$ & 3608 & 768 & 253 & 148 & 47.8 & 5.3 & 4.8 \\
\hline & $\mathrm{CV}^{\mathrm{b}} \%$ & $B L^{a}$ & $22.8 \%$ & $51.5 \%$ & $156.7 \%$ & $222.4 \%$ & $203.1 \%$ & $184.2 \%$ & $163.0 \%$ \\
\hline & Median & $\mathrm{BQL}^{\mathrm{a}}$ & 3739 & 668 & 100 & 21.0 & 7.8 & 2.0 & 1.3 \\
\hline & Range & $\mathrm{BQL}^{\mathrm{a}}$ & 1977 to 4693 & 323 to 1548 & 50.4 to 1215 & 12.2 to 960 & 4.0 to 284 & 1.2 to 29.2 & $\mathrm{BQL}^{\mathrm{a}}$ to 18.9 \\
\hline \multirow[t]{4}{*}{40} & Mean & $B Q L^{a}$ & 7627 & 1838 & 163 & 60.3 & 13.9 & 4.4 & 2.2 \\
\hline & $C V^{b} \%$ & $\mathrm{BQL}^{\mathrm{a}}$ & $36.6 \%$ & $52.2 \%$ & $57.2 \%$ & $50.9 \%$ & $40.3 \%$ & $26.3 \%$ & $53.1 \%$ \\
\hline & Median & $B L^{a}$ & 7194 & 1764 & 156 & 47.9 & 12.9 & 4.1 & 1.8 \\
\hline & Range & $\mathrm{BQL}^{\mathrm{a}}$ & 3782 to 11,792 & 368 to 3349 & 61.5 to 342 & 24.0 to 115 & 7.7 to 25.7 & 3.1 to 6.5 & 1.2 to 4.6 \\
\hline
\end{tabular}

${ }^{a}$ Below quantitative limit.

${ }^{\mathrm{b}}$ Coefficient of variation.

Table 5 Pharmacokinetic parameters following single IV infusion.

\begin{tabular}{|c|c|c|c|c|c|c|c|}
\hline \multirow[b]{2}{*}{ Dose (mg) } & \multirow[b]{2}{*}{ Label } & \multicolumn{6}{|c|}{ Pharmacokinetic parameters } \\
\hline & & $\mathrm{C}_{2-\min }{ }^{\mathrm{a}}(\mathrm{ng} / \mathrm{ml})$ & $\mathrm{AUC}_{0-\infty}{ }^{\mathrm{b}}$ (h-ng/ml) & $\mathrm{Cl}^{\mathrm{c}}\left(\mathrm{ml} / \mathrm{min} / 1.73 \mathrm{~m}^{2}\right)$ & $\mathrm{V}_{\lambda z}{ }^{\mathrm{d}}(\mathrm{l} / \mathrm{kg})$ & $\lambda_{z}{ }^{\mathrm{e}}(1 / \mathrm{h})$ & $t_{1 / 2-\lambda z}{ }^{f}(\mathrm{~h})$ \\
\hline \multirow[t]{4}{*}{10} & Mean & 2676 & 534.1 & 309 & 0.5 & 0.569 & 1.47 \\
\hline & CV\% & $25.58 \%$ & $32.66 \%$ & $26.3 \%$ & $59.3 \%$ & $44.8 \%$ & $49.2 \%$ \\
\hline & Median & 2395 & 485.7 & 297 & 0.4 & 0.595 & 1.18 \\
\hline & Range & 2055 to 3981 & 331.5 to 877.4 & 177 to 442 & 0.2 to 1.2 & 0.275 to 0.955 & 0.73 to 2.52 \\
\hline \multirow[t]{4}{*}{20} & Mean & 3608 & 944.3 & 400 & 0.5 & 0.731 & 1.02 \\
\hline & $\mathrm{CV} \%$ & $22.8 \%$ & $74.0 \%$ & $35.7 \%$ & $48.1 \%$ & $33.9 \%$ & $25.1 \%$ \\
\hline & Median & 3739 & 730.4 & 390 & 0.5 & 0.664 & 1.05 \\
\hline & Range & 1977 to 4693 & 542.1 to 2661.0 & 115 to 626 & 0.1 to 0.9 & 0.522 to 1.293 & 0.54 to 1.33 \\
\hline \multirow[t]{4}{*}{40} & Mean & 7627 & 1490.4 & 470 & 0.7 & 0.560 & 1.31 \\
\hline & CV\% & $36.6 \%$ & $37.7 \%$ & $45.6 \%$ & $50.1 \%$ & $22.6 \%$ & $31.2 \%$ \\
\hline & Median & 7194 & 1451.2 & 412 & 0.5 & 0.551 & 1.26 \\
\hline & Range & 3782 to 11,792 & 641.9 to 2297.8 & 214 to 881 & 0.5 to 1.4 & 0.306 to 0.685 & 1.01 to 2.27 \\
\hline
\end{tabular}

aS-001 plasma concentration at $2 \mathrm{~min}$ from the start of the IV injection.

${ }^{\mathrm{b}}$ Area under the plasma concentration-time curve from time 0 to infinity.

${ }^{\circ}$ Clearance.

dVolume of distribution.

${ }^{\mathrm{e} T e r m i n a l}$ elimination rate constant.

'Elimination half-life. 


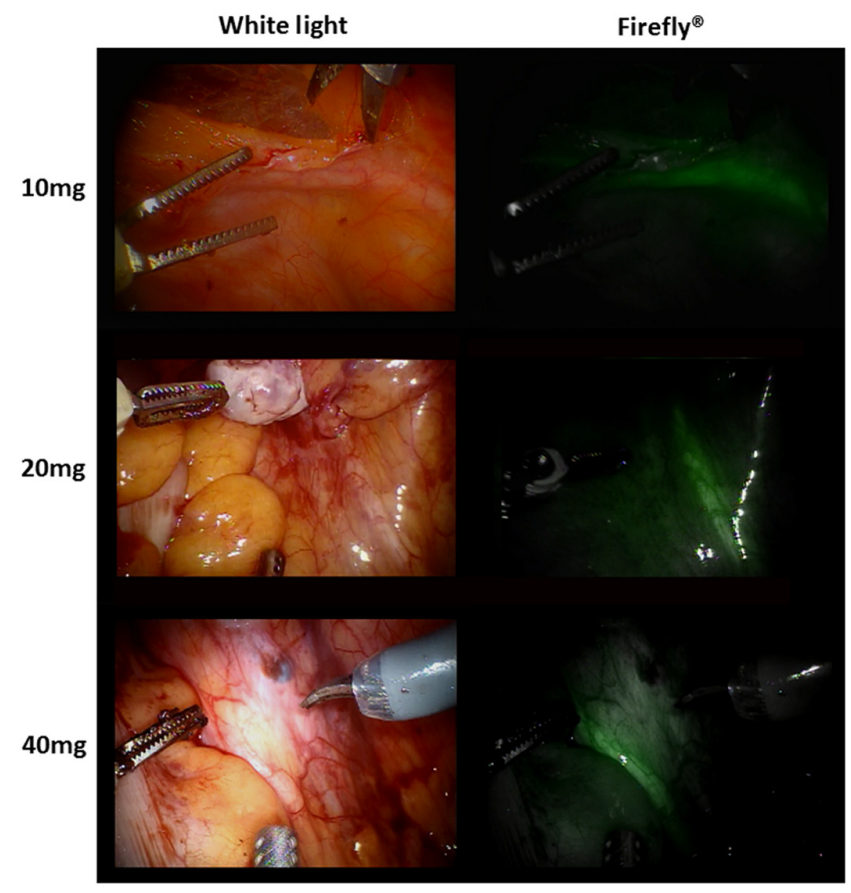

Fig. 2 Ureter near-infrared fluorescence following IV IS-001 injection. Intraoperative white light and near-infrared Firefly ${ }^{\circledR}$ images of the ureter during da Vinci $^{\circledR}$ hysterectomy 20 min after IV IS-001 injection at 10,20 , or $40 \mathrm{mg}$ per patient.

Firefly ${ }^{\circledR}$ mode from all eight subjects $(8 / 8)$ being scored 3 (strong fluorescence), compared with three of eight $(3 / 8)$ in the $20 \mathrm{mg}$ and five of eight (5/8) subjects in the 10-mg dose-cohort (Fig. 3). At 30 min postinjection, ureter images in Firefly ${ }^{\circledR}$ mode from five of eight $(5 / 8)$ subjects in the 40 mg dose-cohort scored 3 (strong fluorescence) relative to one of eight (1/8) in the 20-mg dose-cohort and none of eight (0/ 8 ) in the $10-\mathrm{mg}$ dose-cohort. At the $60 \mathrm{~min}$ postinjection (or last possible time-point if the surgery duration was shorter than $60 \mathrm{~min}$ ) fluorescence intensity was diminished relative to the earlier time-points in all dose-cohorts. Images in Firefly ${ }^{\circledR}$ mode from one of eight (1/8) subjects in the 40-mg dose-cohort scored 3 (strong fluorescence) relative to none of eight $(0 / 8)$ in both the 10- and 20-mg dose-cohorts. However, some fluorescence (a score of 1 or greater) was seen in Firefly ${ }^{\circledR}$ mode images from all eight of eight $(8 / 8)$ subjects in the 40-mg dose-cohort at this time-point, whereas Firefly ${ }^{\circledR}$ mode images from four of eight (4/8) subjects in the 10-mg dose-cohort and five of eight $(5 / 8)$ subjects in the 20-mg dose-cohort scored 0 (no fluorescence) (Fig. 3).

Ureter-to-background signals are shown in Fig. 4. Briefly, representative regions of interest within the ureter and $\sim 5 \mathrm{~cm}$ away from the ureter were used to compute this ratio for all dose cohorts and all time points. This was an exploratory endpoint, and the differences between dose cohorts were not statistically significant.

\section{Discussion}

Iatrogenic ureteral injury remains a severe complication of pelvic surgery that imposes a significant burden in morbidity and health care cost. Approximately 600,000 hysterectomies ${ }^{25}$ and 300,000 colon surgeries ${ }^{26}$ are performed annually in the United States. The estimated ureteral injury rates in hysterectomy and colorectal surgery have been reported anywhere between $0.3 \%$
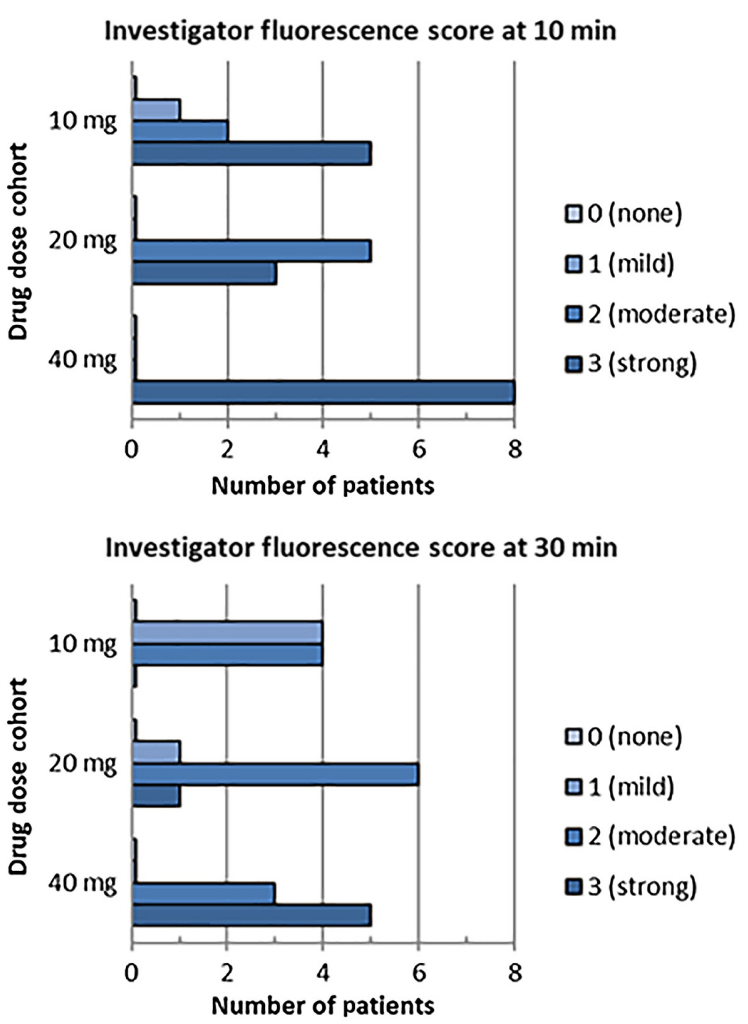

Investigator fluorescence score at $60 \mathrm{~min}$ or last possible time-point

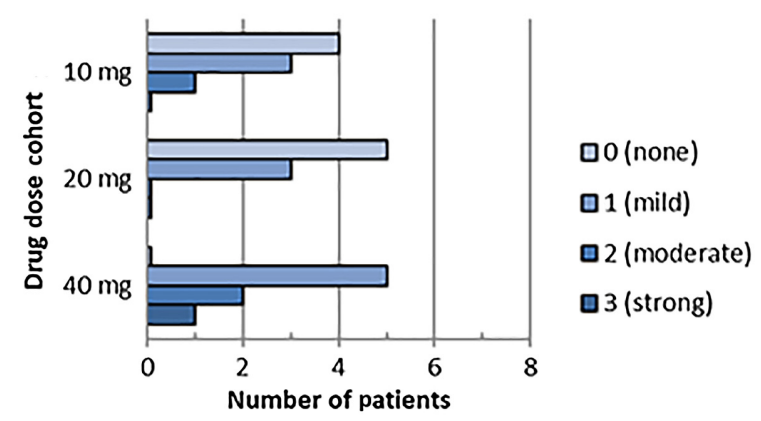

Fig. 3 Intraoperative ureter fluorescence scores. Surgeon-assessed intraoperative ureter fluorescence scores. Assessments were made at 10,30 , and $60 \mathrm{~min}$ (or last possible time-point if surgery lasted less than $60 \mathrm{~min}$ ) postinjection ( $n=8$ for each dose-cohort at each time-point). Images in Firefly ${ }^{\circledR}$ mode scored for ureter fluorescence intensity scored on a 4-point scale from 0 to 3 , where $0=$ none, $1=$ mild, $2=$ moderate, and $3=$ strong fluorescence of the ureter.

to $2.5 \%{ }^{2,27,28}$ and $0.2 \%$ to $7.6 \%$, respectively. ${ }^{29}$ With an average hospital stay of $\sim 4$ days and an average cost of $\$ 31,000$ per ureteral injury, ${ }^{30}$ the economic impact in the United States alone approaches \$1.1B annually.

Avoidance of ureteral injury depends upon clear understanding of anatomic relationships, meticulous surgical technique, and the ability to visually identify the ureter and distinguish it from surrounding structures. Frequently, this requires careful retroperitoneal dissection and surgical ureterolysis. Current methods for intraoperative ureter visualization include ureteral stent placement with palpation, ${ }^{31}$ illuminated catheters, ${ }^{32} \mathrm{x}$-ray fluoroscopy with iodine contrast,${ }^{33}$ or dye injections, ${ }^{34-37}$ techniques that come with significant additional risk to the patient, operating room workflow issues or lack the required sensitivity. 


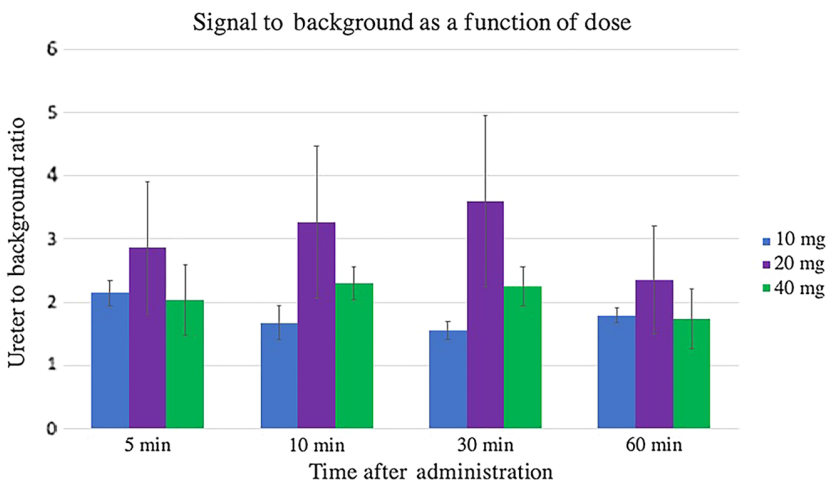

Fig. 4 Green pixels in regions of interest within the ureter and $5 \mathrm{~cm}$ away from the ureter were used to compute a ureter-to-background ratio. While the results were not statistically significant, trends generally indicate a drop off in signal beyond $30 \mathrm{~min}$.

Each of these techniques requires surgical training and privileging beyond the scope of most gynecologic and colorectal surgeons, necessitating intraoperative consultation with urology or urogynecology. A potential advantage of an intravenously administered, renally excreted fluorophore such as IS-001 is that it bypasses this cumbersome requirement, eliminates prolonged operating room delays, and improves surgical workflow.

This first-in-human clinical study supports the safety and tolerability of IV IS-001 injection for fluorescent ureter visualization to doses of up to $40 \mathrm{mg}$ per participant. The four adverse events recorded during this study in two subjects included headache, neck pain, urinary tract infection, and device site pain and were not deemed related to IS-001 (Table 3). All AEs were seen only in the lowest dose cohort $(10 \mathrm{mg})$ and were not observed in the escalating dose-cohorts (20 and $40 \mathrm{mg}$ ).

Similarly, observed changes in laboratory parameters Table $\mathrm{S} 1$ in the Supplementary Materials are consistent with what is reasonably expected after hysterectomy surgery. The increased $\mathrm{WBC}^{19,20}$ and decrease in RBC, $\mathrm{HCT}$ and $\mathrm{HgB},{ }^{21}$ serum albumin, ${ }^{22}$ and serum calcium ${ }^{23}$ is consistent with observed changes following routine surgery and shows no dose-dependent increase in mean change from baseline, suggesting this effect was not drug related. The mean change from baseline normalized over time to the 14-day follow-up time-point. The observed increase in microscopic hematuria noted after surgery is consistent with bladder catheterization and hysterectomy surgery. ${ }^{24}$

The pharmacokinetic analysis shows that IS-001 is rapidly cleared from the blood, limiting unnecessarily prolonged drug exposure when ureter visualization is no longer required, with most subjects reaching the limit of detection of drug in blood plasma by 6-h postinjection (Table 4).

Fluorescent ureter visualization was observed in all subjects following IV infusion of IS-001 when imaged with the $d a$ Vinci ${ }^{\circledR}$ Surgical System's Firefly ${ }^{\circledR}$ fluorescent imaging at all tested doses (Fig. 2). The 40-mg dose-cohort showed the strongest ureter fluorescence at all time-points evaluated postinjection (Fig. 3) when assessed by the operating surgeon.

The intention of this study was to determine the first-inhuman safety and tolerability of IS-001 and establish its pharmacokinetic profile. As a phase I study designed to assess the safety and tolerability of IS-001, this study was not randomized, controlled, or powered to detect differences in ureteral injury at escalating doses. Having detected no drug-related adverse events across all dose cohorts studied and with PK data showing virtually complete elimination at $6 \mathrm{~h}$ at all doses, this study suggests an adjunctive role for IS-001 as a complement to careful surgical technique to facilitate ureteral identification during gynecologic and colorectal surgery. Further study is required to test this hypothesis. The current study looked only at a small group of female patients undergoing straightforward robotic hysterectomy by a single surgeon who rated ureteral fluorescence visualization according to a subjective scale. Participants were overwhelmingly Caucasian, Hispanic, and of middle age, and future studies should expand the demographic scope. Further, the current study considers ureteral visualization only at the pelvic brim, where the ureter can often be seen transperitoneally without use of adjunctive tools to enhance visualization. Future studies should assess ureteral visualization in areas of the pelvis where transperitoneal visualization is not as easily achieved and employ objective means to evaluate intensity of ureteral fluorescence to help elucidate the optimal drug dose and dosing schedule. The current study provides evidence that IV IS-001 shows acceptable early safety and tolerability, provides ureter fluorescence when activated by near-infrared light (Firefly ${ }^{\circledR}$ mode) with higher fluorescence scores at escalating doses. This suggests a potential role for IS-001 in gynecologic and colorectal surgery that future studies designed to account for these limitations can better define.

\section{Disclosures}

Alwin Klaassen disclosed the following-Intuitive Surgical: Employment, ownership interest includes stock, stock options, patent, or other intellectual property. Jonathan Sorger disclosed the following-Intuitive Surgical: Employment, ownership interest includes stock, stock options, patent, or other intellectual property. Richard Farnam-Intuitive Surgical: Proctoring and travel fees. Richard Arms-none.

\section{Acknowledgments}

This study was fully supported by Intuitive Surgical. This study has the clinical trial registration number of NCT03006237 (https://clinicaltrials.gov/ct2/show/NCT03006237).

\section{References}

1. K. Al-Awadi et al., "Iatrogenic ureteric injuries: incidence, aetiological factors and the effect of early management on subsequent outcome," Int. Urol. Nephrol. 37(2), 235-241 (2005).

2. P. Gild et al., "Adult iatrogenic ureteral injury and stricture-incidence and treatment strategies," Asian J. Urol. 5(2), 101-106 (2018).

3. M. D. Barber et al., "Bilateral uterosacral ligament vaginal vault suspension with site-specific endopelvic fascia defect repair for treatment of pelvic organ prolapse," Am. J. Obstet. Gynecol. 183(6), 1402-1411 (2000); discussion 1410-1.

4. J. Tan-Kim et al., "Laparoscopic hysterectomy and urinary tract injury: experience in a health maintenance organization," J. Minimally Invasive Gynecol. 22(7), 1278-1286 (2015).

5. C. J. D. Wallis et al., "Occurrence of and risk factors for urological intervention during benign hysterectomy: analysis of the national surgical quality improvement program database," Urology 97, 66-72 (2016).

6. A. D. Findley and M. J. Solnik, "Prevention and management of urologic injury during gynecologic laparoscopy," Curr. Opin. Obstet. Gynecol. 28, 323-328 (2016).

7. T. E. Nieboer et al., "Surgical approach to hysterectomy for benign gynaecological disease," Cochrane Database Syst. Rev. (8), CD003677 (2015).

8. J. D. Wright et al., "Nationwide trends in the performance of inpatient hysterectomy in the United States NIH public access author manuscript," Obs. Gynecol. 122(1), 233-241 (2013). 
9. T. Parpala-Spårman et al., "Increasing numbers of ureteric injuries after the introduction of laparoscopic surgery," Scand. J. Urol. Nephrol. 42(5), 422-427 (2008).

10. C. Craig, "A particular use for the intravenous injection of indigo carmine," Australas Radiol. 27(3), 273-273 (1983).

11. N. Udshmadshuridze and T. Asikuri, "Intra-operative imaging of the ureter with sodium fluorescein," Z. Urol. Nephrol. 81(10), 635-639 (1988).

12. A. Matsui et al., "Real-time, near-infrared, fluorescence-guided identification of the ureters using methylene blue," Surgery 148(1), 78-86 (2010).

13. M. Al-Taher et al., "Fluorescence ureteral visualization in human laparoscopic colorectal surgery using methylene blue," J. Laparoendosc. Adv. Surg. Tech. 26(11), 870-875 (2016).

14. E. Tanaka et al., "Real-time intraoperative ureteral guidance using invisible near-infrared fluorescence," J. Urol. 178(5), 2197-2202 (2007).

15. R. M. Schols et al., "Application of a new dye for near-infrared fluorescence laparoscopy of the ureters: demonstration in a pig model," Dis. Colon Rectum 57(3), 407-411 (2014).

16. M. L. Korb et al., "Laparoscopic fluorescent visualization of the ureter with intravenous IRDye800CW,' J. Minimally Invasive Gynecol. 22(5), 799-806 (2015).

17. S. M. Mahalingam et al., "Intraoperative ureter visualization using a novel near-infrared fluorescent dye," Mol. Pharm. 15(8), 3442-3447 (2018).

18. J. van den Bos et al., "Near-infrared fluorescence laparoscopy of the ureter with three preclinical dyes in a pig model," Surg. Endosc. 33(3), 986-991 (2019).

19. L. Wijk, K. Nilsson, and O. Ljungqvist, "Randomized control trials metabolic and inflammatory responses and subsequent recovery in robotic versus abdominal hysterectomy: a randomised controlled study," Clin. Nutr. 37(1), 99-106 (2016).

20. M. Goel et al., "Leukocytosis after robotic hysterectomy: commonly observed but clinically insignificant," J. Rob. Surg. 4, 235-239 (2010).

21. S. C. Ribeiro et al., "A randomized study of total abdominal, vaginal and laparoscopic hysterectomy," Int. J. Gynecol. Obstet. 83, 37-43 (2003).

22. S. Mantziari et al., "A novel approach to major surgery: tracking its pathophysiologic footprints," World J. Surg. 39, 2641-2651 (2015).

23. R. Lepage et al., "Hypocalcemia induced during major and minor abdominal surgery in humans," J. Clin. Endocrinol. Metab. 84, 2654-2658 (1999).

24. M. Wilson and H. Merkur, "Hematuria at laparoscopic hysterectomy: a 9-year review at Sydney West Advanced Pelvic Surgery, Australia," J. Minimally Invasive Gynecol. 15, 146-151 (2008).

25. K. M. Doll, S. B. Dusetzina, and W. Robinson, "Trends in inpatient and outpatient hysterectomy and oophorectomy rates among commercially insured women in the United States, 2000-2014," JAMA Surg. 151(9), 876-877 (2016).

26. A. Briggs and J. Goldberg, "Tips, tricks, and technique for laparoscopic colectomy," Clin. Colon. Rectal Surg. 30(2), 130-135 (2017).

27. B. Teeluckdharry, D. Gilmour, and G. Flowerdew, "Urinary tract injury at benign gynecologic surgery and the role of cystoscopy: a systematic review and meta-analysis," Obstet. Gynecol. 126(6), 1161-1169 (2015).
28. P. Härkki-Sirén et al., "Finnish national register of laparoscopic hysterectomies: a review and complications of 1165 operations," Am. J. Obstet. Gynecol. 176(1), 118-122 (1997).

29. G. Silva, M. Boutros, and S. Wexner, "Role of prophylactic ureteric stents in colorectal surgery," Asian J. Endosc. Surg. 5(3), 105-110 (2012).

30. S. N. Zafar et al., "Ureteral injury after laparoscopic versus open colectomy,” JSLS J. Soc. Laparoendosc. Surg. 18(3), e2014.00158 (2014).

31. E. C. Wood, P. Maher, and M. A. Pelosi, "Routine use of ureteric catheters at laparoscopic hysterectomy may cause unnecessary complications," J. Am. Assoc. Gynecol. Laparosc. 3(3), 393-397 (1996).

32. F. Chahin et al., "The implications of lighted ureteral stenting in laparoscopic colectomy," JSLS 6(1), 49-52 (2002).

33. S. Park and M. S. Pearle, "Imaging for percutaneous renal access and management of renal calculi," Urol. Clin. North Am. 33(3), 353-364 (2006).

34. C. F. Jabs and H. P. Drutz, "The role of intraoperative cystoscopy in prolapse and incontinence surgery," Am. J. Obstet. Gynecol. 185(6), 1368-1373 (2001); discussion 1372-3.

35. S. Siddighi, J. J. Yune, and J. Hardesty, "Indocyanine green for intraoperative localization of ureter," Am. J. Obstet. Gynecol. 211(4), 436.e1-436.e2 (2014).

36. Z. Lee et al., "Surgical techniques in urology novel use of indocyanine green for intraoperative, real-time localization of ureteral stenosis during robot-assisted ureteroureterostomy," Urology 82, 729-733 (2013).

37. H. Park and R. W. Farnam, "Novel use of indocyanine green for intraoperative, real-time localization of ureter during robot- assisted excision of endometriosis," J. Minimally Invasive Gynecol. 22, S69 (2015).

Richard W. Farnam is a clinical associate professor at Texas Tech Medical Center and Burrell College of Medicine. He is board certified in urogynecology and is a national thought leader in robotics for reduced port surgery, urogynecology, and advanced technologies. Through his clinical appointments, worldwide presentations, publications, and live tele-surgery, he has trained hundreds of residents, fellows, and practicing surgeons.

Alwin H. Klaassen works in medical imaging at Intuitive Surgical, with a main focus of translating innovative molecular imaging technologies to clinical applications. He has a strong background in molecular biology with a PhD from UCLA in neuroscience and various scientific publications including studies of probe optimization for optical surgical navigation. His professional aims include developing molecular imaging technologies and providing surgeons with better imaging capability to improve surgical safety and outcomes.

Jonathan M. Sorger is the vice president of research at Intuitive Surgical, where he leads a group whose main focus is to address unmet clinical needs through the use of robotic technologies. He received his $\mathrm{PhD}$ and MBA degrees from Johns Hopkins University with a focus on biomedical engineering and magnetic resonance imaging applied to cardiac pathologies.

Biographies of the other author are not available. 